\title{
Reconstructing Images of Bar Codes for Construction Site Object Recognition ${ }^{1}$
}

\author{
by
}

\author{
David E. Gilsinn², Geraldine S. Cheok ${ }^{3}$, Dianne P. O’Leary ${ }^{4}$
}

\begin{abstract}
This paper discusses a general approach to reconstructing ground truth intensity images of bar codes that have been distorted by LADAR optics. The first part of this paper describes the experimental data collection of several bar code images along with experimental estimates of the LADAR beam size and configuration at various distances from the source. Mathematical models of the beam size and configuration were developed and were applied through a convolution process to a simulated set of bar code images similar to the original experiment. This was done in order to estimate beam spread models (beam spread models are unique to each specific LADAR) to be used in a deconvolution process to reconstruct the original bar code images from the distorted images. In the convolution process a distorted image in vector form $g$ is associated with a ground truth image $f$ and each element of $g$ is computed as a weighted average of neighboring elements of $f$ to that associated element. The deconvolution process involves a least squares procedure that approximately solves a matrix equation of the form $H f=g$ where $H$ is a large sparse matrix that is made up of elements from the beam spread function.
\end{abstract}

KEYWORDS: bar codes, deconvolution, image processing, LADAR, object recognition, sparse matrix.

\section{INTRODUCTION}

Imaging sensors such as LADARs (laser distance and ranging devices) are used to rapidly acquire data of a scene to generate $3 \mathrm{D}$ models. They are used to obtain two- or threedimensional arrays of values such as range, intensity, or other characteristics of a scene. Currently available LADARs can gather four pieces of information - range to an object; two spatial angular measurements; and the strength of the returned signal (intensity). Various methods are used to convert the data, which are collected in the form of point clouds, into meaningful 3-D models of the actual environment for visualization and scene interpretation. The points within a point cloud are indistinguishable from each other with regard to their origin; i.e., there is no way to tell if a point is reflected from a tree or from a building. As a result, the methods used to generate the models treat all points identically and the results are indistinguishable "humps/bumps" in the scene. Current surface generation methods using LADAR data require intensive manual intervention to recognize, replace, and/or remove objects within a scene. As a result, aids to object identification have been recognized by the end users as a highly desirable feature and a high priority area of research.

The use of bar codes or UPC (Universal Product Code) symbols has become the universal method for the rapid identification of objects ranging from produce to airplane parts. The same method could also be used to identify objects within a construction scene. This would involve

\footnotetext{
${ }^{1}$ Official contribution of the National Institute of Standards and Technology; not subject to copyright in the United States

${ }^{2}$ National Institute of Standards and Technology (NIST), Information Technology Lab, Mathematical and Computational Sciences Division, Gaithersburg, MD 20899-8910: dgilsinn@nist.gov

${ }^{3}$ NIST, Building and Fire Research Lab, Construction Metrology and Automation Group, Gaithersburg, MD, 20899-8611: cheok@nist.gov

${ }^{4}$ Computer Science Department, University of Maryland, College Park, MD 20742: oleary@nist.gov
} 
using the LADAR to "read" a bar code. The concept is to use the intensity data from the LADAR to distinguish the bar pattern. The advantage of this concept is that no additional hardware or other sensor data is required to gather the additional data. The basis for this concept lies in the high intensity values obtained from highly reflective materials.

The challenges are the ability to read the bar code from $100 \mathrm{~m}$ or greater, distinguish bar code points from the other points in the scene, capture a sufficient number of points to define the bar code for correct identification, and the ability to read bar codes that are skewed.

At ISARC 2001 [1] methods of determining the appropriate material for the bar codes and determining if the use of bar codes was viable were reported. The results from these efforts showed that at distances beyond $20 \mathrm{~m}$, the intensity images were too blurred to be readable and that image processing techniques were necessary to reconstruct the image. The blurring or convolution of the image is a result of the low resolution (number of pixels/unit area; a consequence of the instrument's angular resolution) of the intensity images at larger distances and of distortion of the intensity image by the LADAR optics. As a result, an investigation of possible methods to de-blur (deconvolve) the intensity images was conducted. Deconvolution of the image involves reversing the convolution implying that if the convolution process was known, the image may be reconstructed. The results of the deconvolution study are reported here.

\section{BEAM SIZE AND DIVERGENCE ESTIMATION}

The data for determining the beam size as a function of distance was obtained as part of an experiment to determine the range accuracy of the LADAR as a function of the angle of incidence of the laser beam and distance. An infrared viewer was used to see the projection of the laser on the target so that an outline of the beam could be drawn. The outline of the beam was drawn by two or more observers and the measurements were averaged. The LADAR used consisted of three laser diodes. The projection of the laser beam on the target was seen as a bright rectangle for distances less than $10 \mathrm{~m}$ and three bright vertical bands separated by dark bands for distances greater than $10 \mathrm{~m}$. A schematic of the beam is shown in Figure 1. The measurements are given in Table 1 .

The average vertical beam divergence was 2.14 $\operatorname{mrad}(\sigma=0.39 \mathrm{mrad})$ and the average horizontal beam divergence, excluding an outlier (negative divergence) was $1.86 \mathrm{mrad}(\sigma=0.44 \mathrm{mrad})$. The average beam divergence (horizontal and vertical combined) was $2.01 \mathrm{mrad}(\sigma=$ $0.43 \mathrm{mrad}$ ) - compared with the manufacturer's specified divergence of $3 \mathrm{mrad}$. The lower experimental value was likely a result of the inability of the unaided human eye to detect the faint edges of the laser beam projection. A schematic of the diverging beam is shown in Figure 2.

\section{OPTICS MODEL FOR BEAM DISTORTION}

Discretize the ground truth image by defining: 0 $=y_{1}<y_{2}<\ldots<y_{n f}=1,0=z_{1}<z_{2}<\ldots<z_{n f}=1$, $\Delta y_{i}=y_{i+1}-\mathrm{y}_{\mathrm{i}},=\Delta z_{j}=z_{j+1^{-}} \mathrm{z}_{\mathrm{j} .}=\Delta$. The intensity at patch $\Delta y_{i} \Delta z_{j}$ is given by $\mathrm{f}\left(y_{i}^{*}\right.$, $\left.z_{j}^{*}\right) \Delta y_{i} \Delta z_{j}$ where $f$ is a function of $(y, z)$ expressing the intensity response at some point $\left(y_{i}{ }^{*}, z_{j}{ }^{*}\right)$ in the patch. Due to distortions, the LADAR image of the response from the bar code surface is smeared out into some form of blurred spot shown in Figure 3. The distorted image will be taken as a subset (to be defined below) of the ground truth image for simulation purpose. Points in the distorted image will be identified by $(Y, Z)$ and those in the ground truth image by $(y, z)$. These are different notations for points in the same axis system. The distortion at a point $(Y, Z)$ due to a point $(y, z)$ can be described by a function $h(y, z ; Y, Z)$, called the Beam Spread Function. For most practical purposes, the Beam Spread Function can be considered translation invariant in the sense that its distortion value only depends on the distance between $(Y, Z)$ and $(y, z)$ so that $\mathrm{h}$ has the form 
$h(Y-y, Z-z)$ (see Figure 4). The incremental distortion effect at $(Y, Z)$ due to a neighboring patch of $(y, z)$ is $g(Y, Z)=h(Y-y, Z-z)$ $f(y, z) \Delta y \Delta z$. To describe the total effect $g(Y, Z)$ of all of the points $(y, z)$ in the ground truth image one sums over all of the patches in the ground truth image. As the number of grid points $n f$ in the ground truth image grows and the patch size tends to 0 , the sum can be replaced by an integral. This integral is called a convolution integral.

$$
g(Y, Z)=\int_{-\infty}^{\infty} \int_{-\infty}^{\infty} h(Y-y, Z-z) f(y, z) d y d z
$$

Assume that the distorted image $\mathrm{g}$ is sampled at points $\left(Y_{p}=(p-1) \Delta, Z_{q}=(q-1) \Delta\right)$ for $p, q=$ $1, \ldots, n g$, forming a vector $G$ of $n g^{2}$ elements. Let $h$ be sampled at (ma) $)^{2}$ points $(i \Delta, j \Delta)$ for $i, j$ $=-k,-k+1, \ldots, k-1, k$. This gives a total of $\mathrm{ma}^{2}$ data values $\hat{H}$, where $m a=2 k+1$, and we define all other values of our approximation of $h$ to be zero. Assume that we approximate the ground truth image by $f((i-1) \Delta,(j-1) \Delta) \approx F(i, j)$ for $i, j=1, \ldots, n f$, and again arrange these values as a vector. Then our convolution integral can be approximated by

$$
G(p, q) \approx \sum_{i=1}^{n f} \sum_{j=1}^{n f} \hat{H}(p-i, q-j) F(i, j) \Delta y_{i} \Delta z_{j}
$$

This gives us $n g^{2}$ linear conditions on $n f^{2}$ unknowns $F(i, j)$. Let $H$ be the matrix derived from the values $\hat{H}$ so that our approximation becomes $G \approx H F$.

\section{COMPUTATIONAL ASPECTS FOR IMAGE RESTORATION}

Since the ground truth image $F$ is larger than the distorted image $G$ there are more degrees-offreedom involved in reconstructing $F$ from a measured $G$. A computable approach is to determine $F$ in a least squares manner to satisfy

$$
\min _{F}\|H F-G\| \text {. }
$$

This is an ill-posed problem since there may not be a single solution. In fact it is known that if $F$ is combined with high frequency sinusoidal data, then, under convolution, it produces the same $G$. A penalty term can be added to this minimization problem that puts a premium on the size of $F$ selected. Introduce $\lambda>0$ and form the following minimization problem

$$
\min _{F}\{\|H F-G\|+\lambda\|F\|\}
$$

The second term is called a regularization term and its function is to control the magnitude of the final $F$. In practice, $\lambda$ is selected as a small positive number.

We solve the least squares problem using the LSQR algorithm of Paige and Saunders [2]. This algorithm never modifies elements of $H$ and uses the matrix only to form products $H y$ with various vectors $y$. Thus we need only store the $m a^{2}$ coefficients of $\hat{H}$ rather than the $n g^{2} \mathrm{x}$ $n f^{2}$ matrix $H$.

\section{CONCLUSIONS}

In order to determine the effect of beam spread models on ground truth images, one has to determine the nature of ground truth. The LADAR returns intensity levels in the range 0 to 255. From measured images, it was determined that the intensity of the return signal of the board on which the bars were mounted was approximately 150 and the intensity levels of the bars were approximately 250 . Simulated ground truth data files were created for three sets of barcodes (see ISARC 2001[1]) with an example of $25.4 \mathrm{~mm}$ ( $1 \mathrm{in})$ bars shown in Figure 5. Based upon the measurements of the beam spread function three beam matrices were created to represent the spread function at $10 \mathrm{~m}$, $20 \mathrm{~m}$, and $40 \mathrm{~m}$. Since it was difficult to obtain a precise measurement of the beam spread function, matrices representing the three spatial beam spread configurations were created. They 
were defined in such a manner that the area representing the dark regions was set to zero and the constant value assigned to the light areas was chosen so that the volume under the bright bars summed to unity. With the simulated barcodes and the simulated beam spread functions given, convolution calculations were performed in order to determine how close the simulated distorted images compared to the measured images. The simulated distorted images did not produce the horizontal spread distortion that the measured images produce. Although both the ground truth and beam spread images were simulated it is likely that the lack of prediction was produced by poorly understood beam spread functions. Since the preliminary measurements of the beam spread functions using an infrared scope were crude, this is not surprising. What was surprising, though, was that when the beam spread function at $10 \mathrm{~m}$ was used to deconvolve two images it was possible in a limited fashion to recover the basic details of the ground truth images. In particular, for the case of $25.4 \mathrm{~mm}$ ( 1 in) bars at $10 \mathrm{~m}$, the measured data is given in Figure 6 and shows the lower three bars enmeshed together. The simulated beam spread function for $10 \mathrm{~m}$ was used to recover the ground truth image, and the result is shown in Figure 7. An attempt was then made to reconstruct the ground truth image for the $50.8 \mathrm{~mm}$ (2 in) bars at $10 \mathrm{~m}$ with the same simulated beam spread function. Figure 8 shows that the full reconstruction was not completely obtained. This suggests that the beam spread function might be influenced by the individual image being deconvolved, especially in the presence of noise. It, therefore, is clear that the nature of the beam spread function and its relation to the image being deconvolved is significant. An attempt was then made to construct the beam spread function using the least squares algorithm but in this case, set the matrix $H$ to be the ground truth image and the unknown $F$ to be the unknown beam spread matrix. Figure 9 shows the distorted image created for $(25.4 \mathrm{~mm}(1 \mathrm{in})$ bars using the best fit Beam Spread matrix. It is very close to the actual data measured for the same bars as given in Figure 6. All of the results, though, point to the fact that reconstructing ground truth from distorted LADAR images is critically dependent on knowledge of the Beam Spread Function and how it relates to individual images.

\section{REFERENCES}

1. W. C. Stone, G. S. Cheok, K. M. Furlani, D. E. Gilsinn, "Object Identification Using Bar Codes Based on LADAR Intensity", Proc. of the $18^{\text {th }}$ IAARC / CIB / IEEE / IFAC International Symposium on Automation and Robotics in Construction, ISARC 2001, 1012 September, 2001, Krakow, Poland.

2. C. C. Paige, M. A. Saunders, 'Algorithm 583: LSQR: Sparse Linear Equations and Least Squares Problems'. ACM-Trans. Math. Software, Vol. 8, No. 2, Jun. 1982, pp. 195-209

3. G. Golub, W. Kahan, 'Calculating the singular values and pseudo-inverse of a matrix', J. SIAM Numer. Anal., Ser. B, Vol. 2, No. 2, 1965, pp. 205-224. 


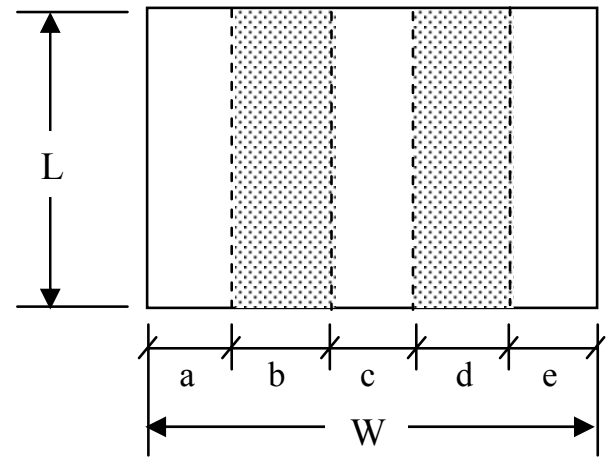

Figure 1. "a", "c" and "e" are the widths of the bright bands and "b" and "d" are the widths of the dark bands.

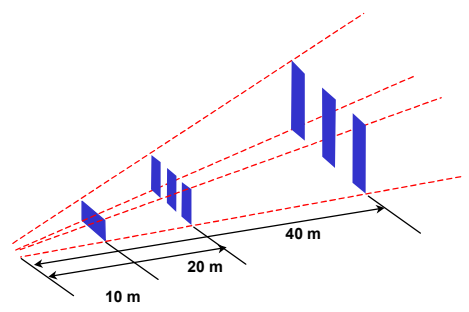

Figure 2. Beam divergence.

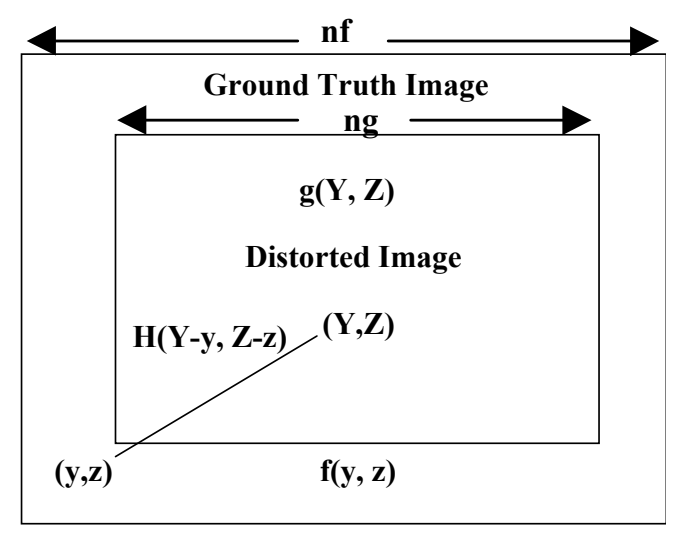

Figure 4. Distorted image overlaid on the ground truth image.

\begin{tabular}{|c|c|c|c|}
\hline & $\mathbf{1 0} \mathbf{~ m}$ & $\mathbf{2 0} \mathbf{~ m}$ & $\mathbf{4 0} \mathbf{~ m}$ \\
\hline $\mathbf{L}$ & 27.5 & 56.2 & 108 \\
\hline $\mathbf{W}$ & 52.4 & 64 & 111 \\
\hline a & - & 12.7 & 15 \\
\hline b & - & 13.2 & 25 \\
\hline c & - & 14.7 & 22.3 \\
\hline d & - & 11.7 & 30.5 \\
\hline e & - & 12 & 18 \\
\hline
\end{tabular}

Table 1. Average height and widths in millimeters of the bright and dark bands of the beam shown in Figure 1.

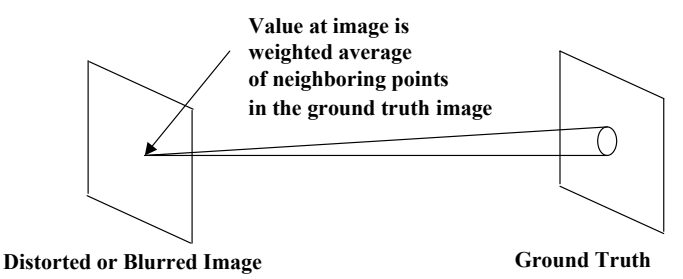

Figure 3. Distortion on the image caused by averaging of pixels in the ground truth image.

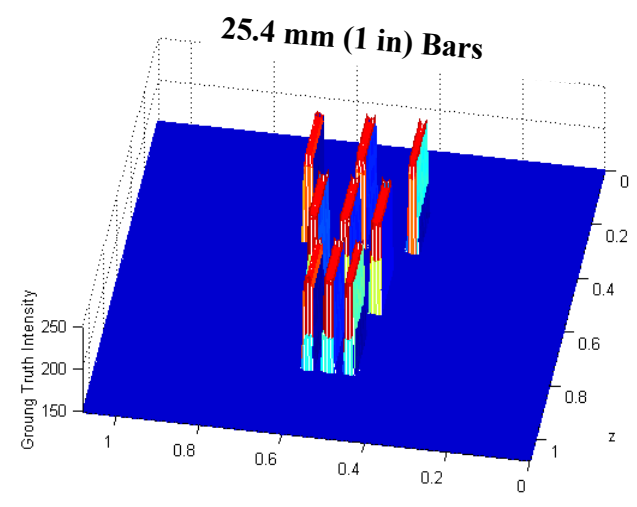

Figure 5. Simulated $25.4 \mathrm{~mm}$ (1 in) barcodes. 


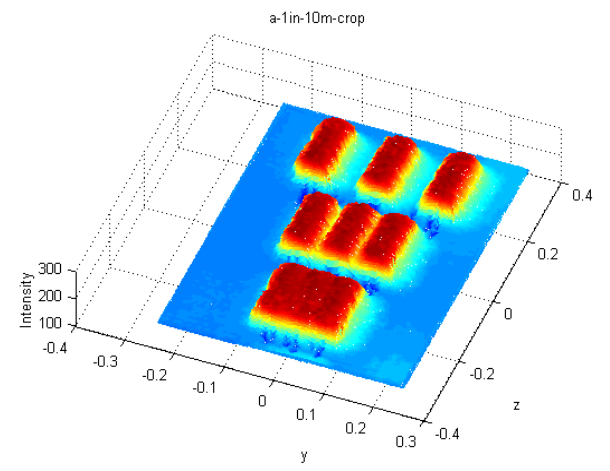

Figure 6. Measured intensity data by LADAR for $25.4 \mathrm{~mm}$ (1 in) bars.

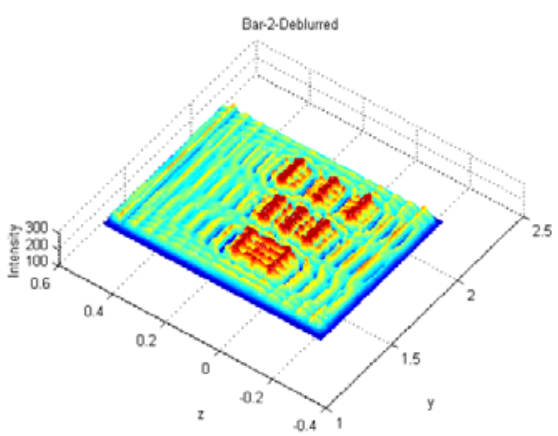

Figure 8. Partial reconstruction of $50.8 \mathrm{~mm}$ (2 in) bars at $10 \mathrm{~m}$.

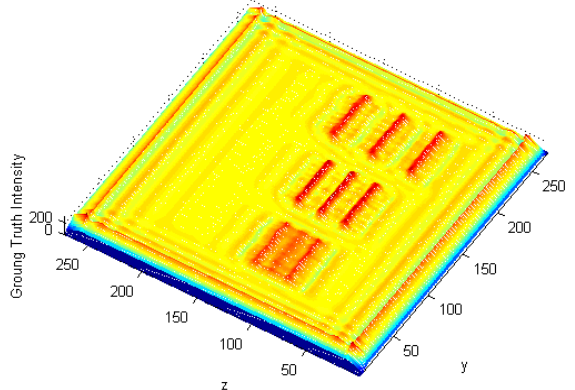

Figure 7. Reconstructed $25.4 \mathrm{~mm}$ (1 in) bars at $10 \mathrm{~m}$.

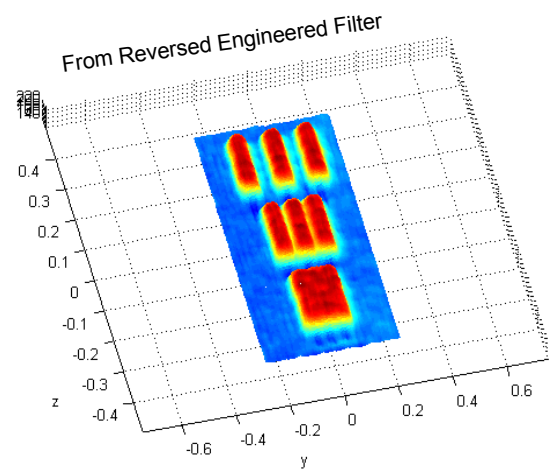

Figure 9. Distorted $25.4 \mathrm{~mm}$ (1 in) bars based on a least squares estimate of the Beam Spread Function. 